\title{
Differential Neural Activity during Search of Specific and General Autobiographical Memories elicited by Musical Cues
}

\author{
Jaclyn Hennessey Ford ${ }^{1}$, Donna Rose Addis², and Kelly S. Giovanello ${ }^{1,3}$ \\ ${ }^{1}$ Department of Psychology, UNC-CH, Chapel Hill, NC, USA ${ }^{2}$ Department of Psychology, The \\ University of Auckland ${ }^{3}$ Biomedical Research Imaging Center, UNC-CH, Chapel Hill, NC, USA
}

\begin{abstract}
Previous neuroimaging studies that have examined autobiographical memory specificity have utilized retrieval cues associated with prior searches of the event, potentially changing the retrieval processes being investigated. In the current study, musical cues were used to naturally elicit memories from multiple levels of specificity (i.e., lifetime period, general event, and eventspecific). Sixteen young adults participated in a neuroimaging study in which they retrieved autobiographical memories associated with musical cues. These musical cues led to the retrieval of highly emotional memories that had low levels of prior retrieval. Retrieval of all autobiographical memory levels was associated with activity in regions in the autobiographical memory network, specifically the ventromedial prefrontal cortex, posterior cingulate, and right medial temporal lobe. Owing to the use of music, memories from varying levels of specificity were retrieved, allowing for comparison of event memory and abstract personal knowledge, as well as comparison of specific and general event memory. Dorsolateral and dorsomedial prefrontal regions were engaged during event retrieval relative to personal knowledge retrieval, and retrieval of specific event memories was associated with increased activity in the bilateral medial temporal lobe and dorsomedial prefrontal cortex relative to retrieval of general event memories. These results suggest that the initial search processes for memories of different specificity levels preferentially engage different components of the autobiographical memory network. The potential underlying causes of these neural differences are discussed.
\end{abstract}

\section{Keywords}

autobiographical memory; medial prefrontal cortex; medial temporal lobe; musical stimuli; memory characteristics; functional MRI

\section{Introduction}

Memory for one's personal past often involves retrieval of abstract, generalized autobiographical knowledge (e.g., "I lived in Chicago in 2006"), as well as specific knowledge of an event that is isolated in place and time (e.g., "I received a purple tricycle at

\footnotetext{
(C) 2011 Elsevier Ltd. All rights reserved.

Address correspondence to: Jaclyn Hennessey Ford, Department of Psychology, University of North Carolina at Chapel Hill, Davie Hall, C.B. \#3270, Chapel Hill, NC 27599.

Publisher's Disclaimer: This is a PDF file of an unedited manuscript that has been accepted for publication. As a service to our customers we are providing this early version of the manuscript. The manuscript will undergo copyediting, typesetting, and review of the resulting proof before it is published in its final citable form. Please note that during the production process errors may be discovered which could affect the content, and all legal disclaimers that apply to the journal pertain.
} 
my $4^{\text {th }}$ birthday party"). Conway and his colleagues (Conway and Pleydell-Pearce, 2000; Conway, Pleydell-Pearce, \& Whitecross, 2001; Conway \& Williams, 2008) have proposed three levels of specificity-lifetime period (i.e., abstract knowledge about oneself during a given period of time), general event (i.e., memory for events that have been repeated or extended beyond one day), and event specific (i.e., memory for an event isolated in place and time). These three levels are believed to exist in a hierarchy of knowledge, with the most abstract personal knowledge (lifetime period) identified as the top level of the system (Conway \& Pleydell-Pearce, 2000).

Although all individuals frequently retrieve both specific and general autobiographical memories (Barsalou, 1988; Conway \& Pleydell-Pearce, 2000), a number of behavioral studies have identified factors that contribute to reductions in specificity. For example, reduced production of specific autobiographical memories has been documented in a number of populations, such as individuals with emotional disorders (see Williams et al., 2007, for review) and healthy older adults (Addis, Wong, \& Schacter, 2008; Levine, Svoboda, Hay, Winocur, \& Moscovitch, 2002; Piolino et al., 2006; Schlagman, Kliegel, Schulz, \& Kvavilashvili, 2009). These groups retrieve more general, and fewer specific, memories relative to healthy young adults.

A majority of research on autobiographical memory specificity has focused on the behavioral characteristics of the memories. However, several researchers have investigated the neural correlates of general and specific autobiographical memory using functional magnetic resonance imaging (fMRI; Addis et al., 2004a; Levine et al., 2004) and positron emission tomography (PET; Maquire \& Mummery, 1999). These studies have shown increased dorsolateral and medial prefrontal activity during retrieval of specific autobiographical information (Levine et al., 2004; Maguire \& Mummery, 1999), suggesting that the prefrontal cortex (PFC) may be essential for successful retrieval or post-retrieval monitoring of a specific autobiographical memory. Increased activity has also been identified in medial temporal lobe (MTL) regions (Levine et al., 2004; Maguire \& Mummery, 1999), and association areas such as the precuneus (Addis et al., 2004a) and retrosplenial cortex (Levine et al., 2004).

In two of these neuroimaging studies, pre-scan interviews were used to collect personal memories from each participant. Maguire and Mummery (1999) collected autobiographical events and facts from each participant and presented the information in the form of "true/ false" propositions in the scanner. Similarly, Addis and colleagues (2004a) collected specific and general autobiographical events from which they created memory titles to present in the scanner. Of note, the use of a pre-scan interview may have introduced a number of confounds by inducing an explicit rehearsal of the event. Providing a personalized memory cue derived from the pre-scan interview eliminates or truncates the search for an autobiographical memory search at test (Cabeza \& St. Jacques, 2007). Therefore, personalized cues may eliminate the search phase of retrieval (i.e., the initial acquisition of the memory), leaving only the elaboration phase (i.e., remembering the event in detail). More importantly, when presented with a personalized cue at test, participants may retrieve the (recent) autobiographical memory of the interview rather than, or in addition to, the original (possibly remote) autobiographical event.

In their comparison of general and specific memories, Levine and colleagues (2004) used a novel prospective method of collecting autobiographical memories of events as they occurred. Participants were asked to record daily events over the course of several months. In the scanner, participants listened to their own 30-second recordings of these personal events. This method allowed researchers to evaluate memory content and accuracy, but did not allow for the analysis of remote memories. Moreover, it is possible that these memories 
may have been encoded differently than typical autobiographical memories due to explicit rehearsal during recording (Cabeza \& St. Jacques, 2007). Finally, participants were presented with all the details of an event during scanning, potentially eliminating the need for an active memory search. As such, the neural differences between memory levels that were identified by Levine and colleagues (2004; discussed above) may not capture differences in the initial search processes.

Although autobiographical knowledge is often described as existing in a hierarchy with three levels of specificity (i.e., lifetime period, general event, and event specific), previous neuroimaging studies have either focused on general event versus event specific memories (Addis et al., 2004a; Levine et al., 2004) or lifetime period versus event specific memories (Maguire \& Mummery, 1999). To better understand the organizational structure of autobiographical memory, research is required that directly examines the neural correlates of all three memory levels. For example, the literature suggests that specific and general event memories are associated with retrieval of event information, with specific memories containing information referring to a particular event and general event information referring to information that extends beyond a single day (Conway \& Pleydell-Pearce, 2000). It is possible that retrieval of these event memories might engage certain neural regions to a greater extent than retrieval of autobiographical knowledge. Moreover, it is likely that the retrieval of temporally specific events may engage additional neural regions above and beyond those engaged by retrieval of general events.

The primary goal of the current study was to compare the neural regions engaged during the initial search process of memories that can be categorized as specific, general, and lifetime period. Raes and colleagues (2007) recently demonstrated that using an unrestricted retrieval prompt produced a distribution of general and specific autobiographical memories without explicit instruction to do so. Other recent studies have suggested that popular music may serve as a unique retrieval cue for this purpose (Cady, Harris, \& Knappenberger, 2008; Janata, 2009; Janata, Tomic, \& Rakowski, 2007). In particular, one fMRI study played musical cues while participants were asked to rate the familiarity, valence, and autobiographical salience of songs (Janata, 2009). In this study, approximately half of the stimuli were rated as being familiar and autobiographical salient, providing a basis for the use of music in autobiographical memory studies in general and, in particular, demonstrating that musical clips can be utilized in the scanner environment to successfully elicit autobiographical memories. Although data about the specific levels of the autographical memories elicited by the musical stimuli were not collected, ratings of the autobiographical salience correlated with activity in medial and ventrolateral PFC. Notably, however, some major nodes of the autobiographical memory network - such as the MTL - were not activated. While this finding contrasts with many others in the field (cf. Svoboda et al., 2006), it may be a result of collapsing across multiple levels of memory. Therefore, the current study extends this prior research by examining the neural regions associated with different levels of autobiographical memories elicited naturally using musical cues.

In order to examine the neural correlates of memory search for specific, general, and lifetime period memory retrieval, we employed a novel paradigm that allowed for retrieval of memories that were highly emotional, unrehearsed, and spanned multiple levels of specificity. Importantly, this method allowed for an analysis of the initial search for information, where previous neuroimaging studies focused largely on the differences in elaboration. We conducted a behavioral pilot study to examine the utility of this musical paradigm. Specifically, we asked fourteen healthy young adults to retrieve autobiographical memories while listening to popular music clips from the past decade. The pilot data indicated that the autobiographical memories retrieved by participants in this study were 
unrehearsed, emotional, and spanned all levels of specificity, thus demonstrating the utility of this paradigm for the current fMRI study.

\section{Methods}

\subsection{Participants}

Sixteen healthy young adults between the ages of 18 and $23(M=20.06 ; S D=1.73$; eight female) participated in the current study. Six additional participants were recruited, but were not included due to early termination of the study (claustrophobia; $n=2$ ), scanner malfunction $(n=2)$, and task non-compliance $(n=2)$. Participants were recruited using flyers posted on the UNC Chapel Hill campus and were paid for their participation. Participants were all right-handed, native English speakers without a history of psychiatric illness, neurological disorder, or hearing impairment. Participants gave written informed consent in accord with the requirements of the Institutional Review Board at the UNC Chapel Hill.

\subsection{Materials}

Retrieval cues for the experimental trials consisted of 30-second clips from popular songs from the years 1998-2007. The top ten songs were selected from each of the ten years using an internet "top ten" website (http://www.rockonthenet.com). Songs were downloaded from the iTunes music store and recorded using MacStim's sound recorder. A 30s clip was selected for each of these 100 songs, and the clip contained the chorus and other highly recognizable segments. Popular songs were selected so that participants would have some level of familiarity with all of the stimuli.

All 100 songs were tested in two pilot studies where 34 undergraduate volunteers listened to the music clips and reported memories associated with each song. Based upon the results of this pilot study, we selected 50 songs that consistently elicited autobiographical memories across all participants. The five songs from each year with the highest ratings of familiarity and memory detail were selected as stimuli for the experimental trials in the current study.

For the control task, seventeen songs were selected to match experimental stimuli in all respects, except song popularity. Selection of control stimuli involved identifying a number of songs from 1998-2007 that matched the experimental stimuli in message, rhythm, and genre. A majority of the control stimuli were songs that never reached popularity, but were from the same albums as our experimental stimuli. Selected control songs were then piloted to test for participant familiarity; any songs that were familiar to even one participant were eliminated from the set of control stimuli.

\subsection{Procedure}

Before scanning, participants engaged in a 30-minute instructional session about the different levels of autobiographical memory. The instruction period was intended to educate participants about the purpose of the study and to ensure that they could effectively rate the characteristics and the level of specificity of each memory. First, participants heard descriptions of the three levels of specificity proposed by Conway and colleagues (lifetime period knowledge, general event knowledge, and event specific knowledge; Conway \& Pleydell-Pearce, 2000). Next, participants were instructed about the rating scales they would use during the experiment (see Appendix).

Once participants had completed the instructions, and felt comfortable with the task, they began the fMRI study. Each participant underwent five consecutive sessions of autobiographical memory retrieval during scanning, followed by a post-retrieval interview outside of the scanner. Each session consisted of ten experimental trials and three or four 
control trials. Pilot testing determined that fifty experimental trials were required to obtain an adequate number of trials in all three levels of specificity.

2.3.1 Experimental Trials-Before each experimental trial, participants were presented with a verbal instruction ("personal") of a jittered duration (2-4s) as a warning to prepare for the upcoming stimulus. Participants listened to 30s clips of popular songs and silently retrieved whatever autobiographical memory came to mind. Importantly, participants were not explicitly instructed to retrieval a specific autobiographical memory, but rather were encouraged to retrieve and elaborate on any memory that came to mind naturally. During each trial, participants identified the level of specificity that best described their memory by pressing the appropriate button (1=lifetime period knowledge, $2=$ general event knowledge, and $3=$ event specific knowledge). If a memory became more or less specific over the duration of the trial, participants acknowledged the change by pressing the corresponding button. As such, some trials were associated with multiple button responses, while others were only associated with one. Participants were asked to keep their eyes closed during the entire task.

Following each musical cue, participants rated two aspects of each memory. The two aspects were randomly selected out of the ten possible rating scales (i.e., familiarity, song preference, genre preference, emotion, intensity, vividness, relivingness, prior rehearsal, relation to previous memory, and recency of memory; see Appendix). For each rating, participants had six seconds to listen to the rating category and select their response with a button press.

2.3.2 Control Trials-Control trials consisted of a $2-4$ s instruction ("adjective") followed by a 30s music clip. For these trials, we used music clips that matched the experimental stimuli in all respects except predicted familiarity for the participants. During the 30 -second clip, participants selected an adjective that described the piece (e.g., optimistic) and, subsequently, generated a definition for the adjective (e.g., "having a positive outlook or view"). After each decision (i.e., after selecting an adjective and after supplying a definition), the participant was asked to select the appropriate button (1=adjective, $2=$ definition). In order to prevent mind-wandering during the control task, participants were asked to focus their attention on the adjective and definition tasks. Following the musical clip, participants rated the song on preference and familiarity.

2.3.3 Post-Retrieval Interview-After completion of the five scanned retrieval runs, participants engaged in a post-retrieval interview in which they were re-presented with the musical cues. During the interview, participants were instructed to recall their previous memory and report it exactly as it had been remembered in the scanner. Participants then rated the memory on all ten characteristics.

\subsection{Data Acquisition}

Magnetic resonance images were acquired using a Siemens Allegra 3-T scanner. Participants' heads were held in place using cushions and a headrest. An initial localizing scan was followed by a high resolution T1 weighted structural scan for anatomical visualization $(1601 \mathrm{~mm}$ slices, $\mathrm{TR}=1750 \mathrm{~ms}, \mathrm{TE}=4.38 \mathrm{~ms}, \mathrm{FOV}=280 \times 320$, Matrix $=224 \times 256$, $1.25 \times 1.25 \times 1.25 \mathrm{~mm}$ resolution). The structural scan was followed by functional scans collected during the five retrieval sessions. Whole brain, gradient-echo, echo planar images $(35 \times 5 \mathrm{~mm}$ slices, TR=2s, TE $=23 \mathrm{~ms}$, Flip angle $=90, \mathrm{FOV}=243$, Matrix $=64 \times 64,3 \times 3 \times 3 \mathrm{~mm}$ resolution) were acquired at an angle perpendicular to the long axis of the hippocampus, identified during the T1 scan. 
To present the stimuli in the scanner, magnet-safe STAX SR-003 headphones were selected to present auditory stimuli with minimal distortion of the auditory signal. Scanner safe noise-reducing earmuffs were used to decrease the amplitude of noise associated with a running scanner. All response data was collected using a magnet-safe button response box.

\subsection{Data Analysis}

Images were preprocessed and analyzed using SPM8 software implemented in MATLAB (Wellcome Department of Cognitive Neurology, London, UK). Images were slice-time corrected, realigned, normalized and smoothed using a Gaussian $8 \mathrm{~mm}$ kernel. Only experimental trials for which the participants successfully retrieved memories (as indicated by at least one button press during the trial and the successful recounting the memory during the post-scan session) were analyzed.

Although we were specifically interested in the neural correlates of the retrieval search phase, we modeled two memory phases within each trial based on RT (Addis et al., 2007); this approach was taken so that the unmodeled elaboration data did not enter into the implicit baseline (Addis, Cheng, Roberts \& Schacter, in press). Memory search was modeled as the two seconds immediately following stimulus onset. For trials where participants identified only one level of memory (and thus only made one button press), the onset of memory elaboration was defined as $1000 \mathrm{~ms}$ before this button press. However, when participants retrieved memories that included multiple levels of memory specificity across the duration of the trial (e.g., a lifetime period cued a general event which then cued event specific knowledge), the multiple button presses delineated a search phase, 1-2 transition phases (between levels of memory), and a final elaboration phase. Still, only two phases were modeled: the search phase of the first level was modeled at cue onset, and elaboration of the final level was modeled $1000 \mathrm{~ms}$ prior to the final button press. The phase(s) between the initial and final button presses was considered a complex mixture of elaboration and search. Because the two processes could not be separated into distinct phases, the mixture was not modeled (Figure 1). Control trials were also modeled as two phases, with control search modeled at cue onset, and control elaboration was modeled $1000 \mathrm{~ms}$ prior to the final button press indicating a definition phase.

Search and elaboration phases for each memory type (lifetime period, general event, and event-specific knowledge) and the control task were entered as regressors into each fixedeffects model. Although both search and elaboration were modelled, the current aims were focused only on the search phase. Thus, contrasts were performed to compare each memory search condition to the control search condition (e.g., Lifetime Period Search > Control Search). Resulting contrast images were entered into a random effects one-way flexible factorial ANOVA with memory level (i.e., lifetime period, general event, and event-specific knowledge) as a single factor. The flexible factorial ANOVA was selected over the full factorial ANOVA to account for subject specific variance (i.e., subject factor), as doing so affords greater sensitivity since the between-subject variance is removed, leaving only the between-task variance. The model was created with a main effect of subject and a main effect of memory level.

The first conjunction analysis examined the neural activity that was common across the three levels of autobiographical memory specificity. This conjunction (i.e., lifetime period knowledge >control and general event>control and event specific >control) allowed us to identify regions associated with all autobiographical memory retrieval, regardless of specificity. We also employed three specific analyses to capture differences between the underlying neural correlates of the autobiographical memory levels. First, we examined neural activity that was greater during retrieval of general event and event specific memory relative to retrieval of lifetime period memory via a conjunction analysis (i.e. general event 
$>$ lifetime period and event specific $>$ lifetime period) with a conjoint probability of $p<$. 001 . This analysis allowed us to identify regions that are preferentially recruited by eventbased (specific and general) autobiographical memories relative to abstract personal knowledge. Another analysis then examined the effect of temporal event specificity by comparing the search of event specific knowledge and general event knowledge (i.e., event specific > general event). Finally, we examined neural activity that corresponded to the continual increase in specificity (i.e., event specific > general event $>$ lifetime period). To confirm that this analysis captured only regions that were significantly greater for event specific than general event and greater for general event than lifetime period, a conjunction analysis was performed (i.e., event specific > general event and general event > lifetime period). For all analyses, a height threshold of $\mathrm{p}<.001$ and an extent threshold of $\mathrm{k}>5$ were used. For visualization purposes, percent signal change was extracted for all three memory types from critical regions identified in each contrast.

\section{Results}

\subsection{Behavioral Results}

Overall, memories were successfully retrieved in $92 \%$ of all trials. Successful memories were categorized based on the initial search level specified by the participant. Importantly, the number of memory searches that began in lifetime period, general, or event specific knowledge did not differ significantly $(F(2,30)=.898 ; \mathrm{p}=.418)$; thus in the fMRI analysis, the three memory-level conditions were equivalent in terms of the number of trials analyzed. Memories identified as lifetime period and general event took an average of 12.24s and 11.76 s to retrieve, respectively. These RTs are similar to the average search time reported in a recent fMRI study that utilized auditory word cues for autobiographical memory search (12.25 s; Daselaar et al, 2007). However, event specific searches were significantly faster than the other two levels $(t(15)=4.495, p<.001$ and $t(15)=2.836, p<.05$ for general event and lifetime period, respectively) at $10.07 \mathrm{~s}$.

The current paradigm permits analysis of how participants transition from one level of retrieval to another. The particular level(s) of memory accessed across the duration of the musical stimulus could differ from one trial to the next, and although the fMRI analysis collapsed across these differences, it is still important to consider the variability across trials. On average, participants remained at one level of memory specificity on a majority of their memory trials $(74.1 \%)$, with most of their other trials (21.6\%) having only one transitional period (i.e., a change from one level to another). Of these transitions, one-third (34.7\%) went from general event to event specific during the retrieval period, twenty-three percent went from lifetime period to general event, fourteen percent went from lifetime period to event specific, and sixteen percent went from event specific to general event.

The musical cuing paradigm was specifically designed to elicit retrieval of highly emotional memories from all three memory levels. Participants rated 17\% of memories as highly negative and $17.5 \%$ as highly positive. Additionally, we asked participants for a "prior rehearsal" rating that measured the degree of previous search for each memory. For all three memory levels, this level was very low $(1.53,1.49$, and 1.52 out of 4 for lifetime period, general event, and event specific), indicating that the retrieved memories had low levels of rehearsal. As such, this paradigm allowed for the examination neural activity associated with memories that had not bee retrieved multiple times previously.

The memory characteristics ratings that were obtained during the post-retrieval interview were compared across the three levels of autobiographical memories. Table 1 summarizes these behavioral data. Event specific memories were significantly more emotionally intense, vivid, and re-experienced than general event memories $(t(14)=2.731, p<.05 ; t(14)=3.996$, 
$p<.001$; and $t(14)=3.972, p<.001)$ and more positive, vivid, and re-experienced relative to lifetime period memories $(t(14)=3.185, p<.01 ; t(14)=4.976, p<.001$; and $t(14)=4.469, p<$. $001)$. General event memories were rated as more positive, vivid, and re-experienced than lifetime period memories $(t(14)=2.323, p<.05 ; t(14)=2.405, p<.05 ; t(14)=2.752, p<.05)$.

Highly familiar songs were required to obtain memories from a range of levels without providing explicit instructions to do so. Familiarity of songs was high across all three conditions (rated 3.14, 3.46, and 3.59 out of 4 for lifetime period, general event, and eventspecific, respectively) and was significantly higher than in the control condition $(t(14)=$ 11.29, $p$ <.001: $t(14)=16.13, p<.001$ : and $t(14)=18.49, p<.001$ for lifetime period, general event, and event specific, respectively) at an average of 1.24 out of 4.

\subsection{Imaging Results}

\subsubsection{Common neural activity during search of all autobiographical memories}

-A conjunction analysis was performed to identify neural regions recruited by retrieval of all three types of autobiographical memory (i.e., lifetime period, general event, and event specific knowledge) relative to the control condition1. This comparison revealed regions that were commonly activated during retrieval of all memory types, regardless of the level of specificity. A number of regions that have consistently been reported in autobiographical memory studies were identified in this conjunction analysis: Bilateral activation in the posterior cingulate, lateral temporal lobes, and parahippocampal gyrus, extending into the right hippocampus. Additionally, activity in the ventromedial PFC was left lateralized (see Table 2 and Figure 2).

\subsubsection{Neural activity associated with the search for autobiographical event memories versus autobiographical knowledge-The current analysis examined} differences in neural regions engaged during search for memories categorized as event information relative to those classified as abstract personal knowledge2. To this end, a conjunction analysis was performed that identified activity associated with event specific and general event information to a greater extent than lifetime period knowledge (i.e., event specific > lifetime period and general event $>$ lifetime period). In this contrast we identified bilateral activity in dorsomedial PFC, dorsolateral PFC, posterior cingulate, and lateral temporal lobes. In addition, a small cluster of significant activity was observed within the right MTL (see Table 3 and Figure 3).

\subsubsection{Neural activity associated with the search for temporally specific} memories-We also directly compared neural regions engaged during the search for memories classified as general events and those classified as specific events (i.e. event specific > general event). This comparison examined regions that were preferentially involved in temporally specific events relative to extended or categorical events. The event specific memory condition was associated with increased activity in a number of regions in the autobiographical memory network. Of particular interest, we identified bilateral activity in the MTL (including bilateral parahippocampal gyrus and the left hippocampus), medial PFC, dorsolateral PFC, posterior cingulate, lateral temporal lobes, and precuneus (See Table 4 and Figure 4). Additionally, this contrast showed bilateral activity in the superior parietal lobes3.

\footnotetext{
${ }^{1}$ We also performed a conjunction analysis in which we split the control condition into three subsets, for use as independent baselines for the three conditions of interest (Addis et al., in press). The regions of significance in this conjunction were identical to those reported in this paper.

${ }^{2}$ Of note, the current paradigm does not instruct participants as to which level they should retrieve. As such, their search is not a controlled search for a particular level, but rather a more general search for a memory that can then be classified into a level.
} 


\subsubsection{Neural activity associated with a continual increase in memory}

specificity-A conjunction analysis examined activity corresponding to continual increase in specificity from lifetime period knowledge to event specific memory (i.e. event specific > general event and general event > lifetime period). This contrast identified only those regions associated with an increase in activity from lifetime period to general event, as well as an increase in activity from general event to event specific. This contrast revealed a network of frontal activations that was largely left lateralized. Specifically, we identified activity in the dorsolateral and dorsomedial PFC, in regions similar to those highlighted in previous contrasts (See Table 5 and Figure 5). This contrast tracked with phenomenological ratings (i.e., emotional intensity, valence, reliving, and vividness) that also showed continual increases across the three memory levels, suggesting that increasing frontal activity during retrieval is associated with increasingly rich memories.

\section{Discussion}

The current study used musical cues to examine the commonalities and differences in the neural underpinnings of three levels of autobiographical memory specificity. In particular, we found that certain neural regions (e.g., ventromedial PFC, MTL, and posterior cingulate) were recruited during search of all autobiographical memory conditions, whereas other regions were preferentially engaged during retrieval of memories classified as event-based irrespective of temporal specificity (e.g., dorsolateral and dorsomedial PFC) or temporallyspecific events (e.g., dorsomedial PFC, bilateral MTL, and inferior frontal gyrus). Moreover, this research serves as further support for the use of music as a retrieval cue in autobiographical memory tasks.

\subsection{Neural Activation during Search for All Autobiographical Memories}

Previous studies of autobiographical memory specificity have used highly structured retrieval paradigms with pre-generated cues that circumvent the need for an effortful memory search; as such, these studies have focused solely on the neural correlates of direct retrieval and elaboration of autobiographical memory. Expanding on these previous studies, the use of non-personal musical cues in the current design had the unique ability to examine neural activity during the early search phase of retrieval of memories that ranged across three levels of specificity: lifetime period, general event, and event specific memories.

The conjunction analysis examining retrieval of all three memory levels demonstrated that, regardless of specificity, the search for an autobiographical memory engages key regions of the core autobiographical memory network. This network included bilateral regions of the posterior cingulate, lateral temporal lobes, and ventromedial PFC. Importantly, neuroimaging studies have associated activity in the ventromedial PFC with self-reference during autobiographical memory tasks (see Cabeza \& St. Jacques, 2007) and the posterior cingulate with integrating self-referential stimuli in the autobiographical context (Fink et al. 1996; for review, see Northoff and Bermpohl 2004). In addition, all memory conditions were associated with bilateral activity in the MTL, a region of vital importance to autobiographical memory retrieval (Addis et al., 2004b; Addis and Schacter, 2008; Ford, Addis, \& Giovanello, under review; Gilboa, Winocur, Grady, Hevenor, \& Moscovitch, 2004; Markowitsch et al., 2003; Piefke et al., 2003; Rabin, Gilboa, Stuss, Mar, \& Rosenbaum, 2009; Viard et al., 2007). Overall, the regions typically associated with autobiographical memory were engaged during the early stages of all autobiographical

\footnotetext{
${ }^{3}$ To distinguish this contrast from the subsequent contrast examining the continual increase in activity across specificity, we also ran this analysis using an exclusive mask of the continual contrast. This new analysis identified identical peak voxels and neural regions as those observed in the unmasked contrasts, with minimal reduction in the size of some clusters.
} 
memory retrieval, regardless of the episodic specificity of the information ultimately retrieved.

Many of the regions observed in the conjunction analysis have been identified previously as belonging to a core autobiographical memory retrieval network - a group of regions consistently reported in autobiographical memory studies (Svoboda, McKinnon, \& Levine, 2006). In addition, the current results are broadly consistent with the few studies that have focused on the neural correlates of autobiographical memory specificity (Addis et al, 2004a; Maguire \& Mummery, 1999). For example, Addis and colleagues (2004a) used spatiotemporal partial least squares (PLS) to identify a network of regions commonly activated by both specific and general event memories, compared to a semantic control task. Although these memories had been previously retrieved during a pre-scan interview, the neural network engaged during that study was similar to the network reported here, including left posterior cingulate, left inferior temporal gyrus, and bilateral parahippocampus and hippocampus. The current study expanded upon these previous findings in several ways. Here, we showed that the recruitment of the core autobiographical network previously observed during retrieval was also engaged during the search for memories. Moreover, we show that this network is activated not only for event (general and specific) memories, but also activated to some degree during search for the more abstract representations of lifetime periods.

The ability to analyze trials from all three levels of autobiographical memory enabled us to examine how specificity modulated activation of the core autobiographical memory network. The contrast comparing general event and event specific retrieval to that of lifetime period retrieval was particularly interesting because it highlighted regions that were engaged by retrieval of events (both general and specific) to a greater extent than those engaged by retrieval of abstract personal knowledge. Such regions included medial and lateral PFC, MTL, and lateral temporal lobe activity. Our results are consistent with a study that has compared one class of event memory (i.e., specific) with autobiographical knowledge (Maguire and Mummery, 1999), in which the authors found activation of medial PFC, hippocampus, and lateral/polar temporal cortex.

Interestingly, although the current analysis identified a number of regions (e.g., dorsolateral and dorsomedial PFC) as being differentially active for both types of event memories (relative to autobiographical knowledge), examination of the percent signal change in these regions revealed that activation across these two event conditions (i.e., event specific and general event) was not equivalent. Retrieval of specific events engaged these regions more than general events. This apparent difference was confirmed by an analysis that looked for continual increases in activation that tracked increases in specificity. Our analysis of these dorsal frontal regions revealed that activity during retrieval of lifetime period memories was reduced relative to the active baseline. Similarly, we found no difference between general event memories and the control condition. As the control task required intentional, controlled search processes, it is not entirely surprising that we identified some level of activation in these PFC regions during this task. As dorsolateral PFC regions have been implicated in controlled retrieval processes (Conway et al., 1999; Maguire et al., 2001; Steinvorth et al., 2006; Svoboda et al., 2006), this finding suggests that retrieval of general memories (i.e., lifetime period and general event) may occur when individuals do not actively engage in controlled retrieval of mnemonic details. In fact, specific events were rated as high in vividness and re-experiencing. Additionally, the retrieval of increased detail in specific event memories may require additional post-retrieval monitoring of this content, also engaging dorsolateral PFC (e.g., Hayama and Rugg, 2009). However, the current study did not manipulate these variables directly to experimentally test for associated changes. 
In another analysis, specific events were directly compared to general events. Many of the regions that were preferentially engaged by specific, relative to general, event retrieval were not identified in the continual contrast. That is, activity in these regions did not simply track with an increase in specificity, but was unique to specific events. The differences between specific and general event retrieval identified here are consistent with the findings from other studies, despite the fact those studies collected memories prior to scanning and thus did not differentiate between search for, and the subsequent elaboration of, specific event memories. Addis and colleagues (2004a) identified regions associated with imagery in autobiographical memory (i.e., left precuneus, left superior parietal lobe, and right cuneus), while Levine and colleagues (2004) identified regions associated with self reference (i.e., left anteromedial PFC) as being differentially activated by specific events. These regions were also observed in the current analysis. However, by focusing on the early search phase of event specific information, we also identified differential activity in other regions considered critical to autobiographical memory retrieval, such as the bilateral MTL and lateral temporal lobes. It is notable that specific memories are associated with increased activity in the bilateral MTL, as recent research has demonstrated that hippocampal activity is modulated by vividness (Addis et al., 2004b; Addis and Schacter, 2008; Ford, Addis, \& Giovanello, under review; Gilboa, Winocur, Grady, Hevenor, \& Moscovitch, 2004; Rabin, Gilboa, Stuss, Mar, \& Rosenbaum, 2009; Viard et al., 2007), emotion (Ford, Addis, \& Giovanello, under review; Markowitsch et al., 2003; Piefke et al., 2003; Viard et al., 2007), and reliving of events (Viard et al., 2007). Specific memories in this study were more vivid, emotionally intense, and subject to higher levels of to reliving than general event memories, suggesting that these aspects of memory contributed to the increased MTL activity.

Although the current study does not directly examine retrieval strategies, it is possible that distinct retrieval processes may underlie retrieval of specific and general memories. Because specific events are accessed significantly more quickly, it is possible that this is a form of direct rather than generative autobiographical memory retrieval (Conway \& PleydellPearce, 2000; Haque and Conway, 2001). In these cases, the musical cue may be so closely associated with a particular memory that it is essentially a personalized cue. Indeed, Conway and colleagues (Conway \& Pleydell-Pearce, 2000; Haque and Conway, 2001) have suggested that highly salient and personal memory cues may facilitate direct retrieval of the event specific details linked to an autobiographical memory. Musical cues that prove particularly salient to an individual may allow one to retrieve a more complex and detailed memory representation, resulting in more activity in medial temporal regions (Addis et al., 2004b). As such, musical cues have the potential to be ideal stimuli for further research examining the differences between the neural correlates of generative and direct autobiographical memory retrieval.

\subsection{Music and Autobiographical Memory}

A secondary goal of the current experiment was to evaluate the utility of music as a cue in autobiographical memory studies. Music has been proposed as an emotional and selfrelevant memory cue that can be used universally across participants of the same cohort (Cady et al., 2008; Janata et al., 2007). Unlike the songs used in previous studies, the materials used in the current study were all highly familiar to participants, yielding a high number of successfully retrieved memories. Additionally, the current study took advantage of music's ability to cue different memory levels of autobiographical memory specificity, and examined how patterns of neural activity differed across these different memory levels. Using musical cues also allowed participants to retrieve a wide range of memories that had not been retrieved many times previously. Accessing relatively under-rehearsed memories gives this study an advantage over neuroimaging studies that have employed a pre-scan 
interview (e.g. Addis et al., 2004a) or prospective collection of memories (e.g. Levine et al., 2004).

One previous neuroimaging study has evaluated autobiographical memories during the presentation of musical cues (Janata, 2009). In this study, participants were asked to listen to musical cues and evaluate the familiarity, valence, and autobiographical salience of each song. As Janata (2009) selected songs that varied in their familiarity to participants, his study examined regions engaged during presentation of familiar and autobiographically salient songs versus those that lacked these qualities. Although not explicitly instructed to retrieve memories in response to the musical cues, participants rated the autobiographical salience of each song ( $1=$ no association, $2=$ weak association, $3=$ strong association; Janata, 2009). The data indicated that $42 \%$ of songs had some level of salience and were thus likely accompanied by spontaneous retrieval of autobiographical memories. Indeed, in a post scan interview, Janata (2009) confirmed that songs rated as having a stronger autobiographical association were associated with more vivid remembering of emotion-laden memories of events. This confirmed previous research from the same lab suggesting that increased ratings of autobiographical salience are associated with a greater rate of spontaneous memory retrieval during song presentation (Janata et al., 2007). Importantly, these studies suggest that music may lead to retrieval of memories, even in the absence of retrieval instructions.

Janata's (2009) analysis revealed a small set of regions whose activation was modulated by autobiographical salience, and interestingly, some of these regions were also evident in the current study. In particular, ventromedial PFC activity was evident across all three memory levels, which is consistent with the fact that all of the memories in the current study were autobiographical and self-referential in nature (Cabeza \& St. Jacques, 2007). Moreover, the prefrontal regions that Janata (2009) identified as modulating with salience (ventromedial, dorsomedial and ventrolateral cortex) were, in this study, more active with the increasing specificity of the memories. This finding may reflect some overlap between the subjective experience of autobiographical salience that is afforded by the richness of specific memories.

Interestingly, the measures of autobiographical salience reported by Janata (2009) did not correspond to the activity in the MTL or dorsolateral PFC identified in the current study. These differences may arise for a number of reasons. First, Janata examined activity during the entire 30-second music clip presentation, whereas the current paper focused on the early search phase of retrieval. It is conceivable that collapsing across this entire period obscured activation in regions that are not consistently active across the trial. For instance, Daselaar et al. (2008) found that while medial temporal and dorsolateral regions were active during the initial search and access of autobiographical memories, other regions, including ventrolateral and ventromedial regions identified by Janata, exhibited a more sustained timecourse throughout elaboration. Moreover, collapsing across trial-types with different activation profiles could also obscure activity. In this study, we found that medial temporal regions showed increasing activity with increasing specificity of events, and the left hippocampus was most strongly and extensively activated during the retrieval of temporally specific events. Janata (2009) did not report the specificity of the autobiographical memories retrieved during retrieval, but the lack of hippocampal activity in that study would suggest, on the basis of the current findings, there was a predominance of non-specific memories.

It is also possible that there are neural differences between the intentional search for autobiographical memories, and the spontaneous access of memories to highly familiar and salient cues. As Janata (2009) did not explicitly instruct subjects to retrieve memories in response to the musical cues, memory retrieval in this case could be considered spontaneous and involuntary, and may underlie the lower rates of trials that resulted in autobiographical 
associations (42\%, as compared to $92 \%$ in the current study). Little is known about the neural basis of involuntary versus intentional autobiographical retrieval. Comparison of these two studies could lead to the speculation that activation in the MTL is particularly evident with intentional retrieval conditions, such as in the current study. Interestingly, a recent study found hippocampal activity associated with intentional versus incidental retrieval of explicit memories (Ramponi, Barnard, Kherif, \& Henson, 2011), suggesting that such an effect may be evident for autobiographical memory retrieval. However, further research that addresses this question directly is required.

\subsection{Summary}

The current study used unrestricted retrieval of autobiographical memories to identify characteristics and neural activation differences between three levels of autobiographical memory. Behavioral data revealed that participants rated event specific memories as the most vivid, emotional, positive, and subject to reliving. Additionally, these memories were associated with increased activation in several regions within the core network, such as the left MTL and dorsomedial PFC. However, other regions within the core network, such as the ventromedial PFC and posterior cingulate, were engaged during all autobiographical memory tasks.

The results of this study also support recent studies that introduce musical stimuli as ideal cues for autobiographical memory tasks (Cady et al, 2008; Janata, 2009; Janata et al, 2007) and expand on this research by demonstrating the value of a musical cue paradigm for investigating questions about the theoretical structure of intentional autobiographical memory. Musical cues allowed participants in the current study to retrieve all three levels of autobiographical memory naturally, without explicit instruction. Additionally, the memories retrieved were often highly emotional (both negative and positive) and had low levels of prior retrieval. These findings support the use of musical cues in future studies of autobiographical memory, particularly those in populations such as depressed individuals and healthy older adults, who may experience difficulty retrieving specific memories.

Highlights

- Young adults retrieved autobiographical memories associated with musical cues

- Music-cued memories spanned three levels of specificity, without explicit instruction

- Music-cued memories were highly emotional and had low levels of prior rehearsal

- Specific memories were associated with increased activity in dorsal frontal regions

- All autobiographical memories engaged medial frontal and temporal regions

\section{Acknowledgments}

This work was supported by NIA AG028774 to KSG.

\section{References}

Addis DR, Cheng T, Roberts RP, Schacter DL. Hippocampal contributions to the episodic simulation of specific and general future events. Hippocampus. (in press). 
Addis DR, McIntosh AR, Moscovitch M, Crawley AP, McAndrews MP. Characterizing spatial and temporal features of autobiographical memory retrieval networks: A partial least squares approach. NeuroImage. 2004a; 23:1460-1471. [PubMed: 15589110]

Addis DR, Moscovitch M, Crawley AP, McAndrews MP. Recollective qualities modulate hippocampus activation during autobiographical memory retrieval. Hippocampus. 2004b; 14:752762. [PubMed: 15318333]

Addis DR, Wong AT, Schacter DL. Remembering the past and imagining the future: Common and distinct neural substrates during event search and elaboration. Neuropsychologia. 2007; 45:13631377. [PubMed: 17126370]

Addis DR, Wong AT, Schacter DL. Age-related changes in the episodic simulation of future events. Psychological Science. 2008; 19(1):33-41. [PubMed: 18181789]

Barsalou, LW. The content and organization of autobiographical memories. In: Neisser, U.; Winograd, E., editors. Remembering reconsidered: Ecological and traditional approaches to the study of memory. New York, NY: Cambridge University Press; 1988. p. 193-243.

Cabeza R, Jacques St. Functional neuroimaging of autobiographical memory. Trends in Cognitive Sciences. 2007; 11(5):219-227. [PubMed: 17382578]

Cady ET, Harris RJ, Knappenberger JB. Using music to cue autobiographical memories of different lifetime periods. Psychology of Music. 2008; 36:157-177.

Conway MA, Pleydell-Pearce CW. The search of autobiographical memories in the self-memory system. Psychological Review. 2000; 107:261-288. [PubMed: 10789197]

Conway MA, Pleydell-Pearce CW, Whitecross SE. The neuroanatomy of autobiographical memory: A slow cortical potential study of autobiographical memory retrieval. Journal of Memory and Language. 2001; 45:493-524.

Conway, MA.; Williams, HL. Autobiographical Memory. In: Roediger, HL., III, editor. Learning and Memory: A Comprehensive Reference, Vol. 2: Cognitive Psychology of Memory. New York: Elsevier; 2008. p. 893-910.

Daselaar SM, Rice HJ, Greenberg DL, Cabeza R, LaBar KS, Rubin DC. The spatiotemporal dynamics of autobiographical memory: Neural correlates of recall, emotional intensity, and reliving. Cerebral Cortex. 2008; 18:217-229. [PubMed: 17548799]

Gilboa A, Winocur G, Grady CL, Hevenor SJ, Moscovitch M. Remembering our past: Functional neuroanatomy of recollection of recent and very remote personal events. Cerebral Cortex. 2004; 14:1214-1225. [PubMed: 15166099]

Haque S, Conway MA. Sampling the process of autobiographical memory search. European Journal of Cognitive Psychology. 2001; 13:529-547.

Hayama HR, Rugg MD. Right dorsolateral prefrontal cortex is engaged during post-retrieval processing of both episodic and semantic information. Neuropsychologia. 2009; 47(12) 2409-1416.

Janata P. The neural architecture of music-evoked autobiographical memories. Cerebral Cortex. 19(11):2579-3594.

Janata P, Tomic ST, Rakowski SK. Characterization of music-evoked autobiographical memories. Memory. 2007; 15(8):845-860. [PubMed: 17965981]

Levine B, Svoboda E, Hay JF, Winocur G, Moscovitch M. Aging and autobiographical memory: Dissociating episodic from semantic retrieval. Psychology and Aging. 2002; 17(4):677-689. [PubMed: 12507363]

Levine B, Turner GR, Tisserand D, Hevenor SJ, Graham SJ, McIntosh AR. The functional neuroanatomy of episodic and semantic autobiographical remembering: A prospective functional MRI study. Journal of Cognitive Neuroscience. 2004; 16:1633-1646. [PubMed: 15601525]

Maguire EA, Mummery CJ. Differential modulation of a common memory retrieval network revealed by positron emission tomography. Hippocampus. 1999; 9:54-61. [PubMed: 10088900]

Piolino P, Desgranges B, Clarys D, Guillery-Girard B, Taconnat L, Isingrini M, et al. Autobiographical memory, autonoetic consciousness, and self-perspective in aging. Psychology and Aging. 2006; 21(3):510-525. [PubMed: 16953713] 
Rabin JS, Gilboa A, Stuss DT, Mar RA, Rosenbaum RS. Common and unique neural correlates of autobiographical memory and theory of mind. Journal of Cognitive Neuroscience. 2009; 22:10951111. [PubMed: 19803685]

Raes F, Hermans D, Williams JM, Eelen P. A sentence completion procedure as an alternative to the Autobiographical Memory Test for assessing overgeneral memory in non-clinical populations. Memory. 2007; 15(5):495-507. [PubMed: 17613793]

Ramponi C, Barnard PJ, Kherif F, Henson RN. Voluntary explicit versus involuntary conceptual memory are associated with dissociable fMRI responses in hippocampus, amygdala, and parietal cortex for emotional and neutral word pairs. Journal of Cognitive Neuroscience. 2010

Schlagman S, Kliegel M, Schulz J, Kvavilashvili L. Differential effects of age on involuntary and voluntary autobiographical memory. Psycholology and Aging. 2009; 24(2):397-411.

Tulving, E. Elements of episodic memory. Vol. 2. New York, NY: Oxford University Press; 1983.

Williams JM, Barnhofer T, Crane C, Herman D, Raes F, Watkins E, et al. Autobiographical memory specificity and emotional disorder. Psychological Bulletin. 2007; 133(1):122-148. [PubMed: 17201573]

\section{Appendix}

\section{Instructions for Memory Ratings}

Familiarity: How familiar are you with the song? 1= not at all, 4= know it almost perfectly

Song Preference: How much do you like the song? 1= not at all, 4= one of your favorites

Genre Preference: How much do you like songs like the one you just listened to? 1= not at all, 4= one of your favorites

Emotion/Affect: How negative or positive was the memory? 1= highly negative, 4= highly positive

Intensity: How intense (emotionally) was this memory? 1= not at all, 4= very

Vividness: How many details can you retrieve about this memory? How clear are these details? 1= very vague memory, $4=$ very clear and distinct

Reliving: Can you put yourself back into the memory? 1= almost like watching the events unfold like a home movie, $4=$ a feeling of re-experiencing the event

Prior Search: When was the last time you retrieved (remembered) this event? 1= haven't really thought about the event since it happened, $4=$ I have retrieved this event very recently

Relation: How strong is the relation between the memory you retrieved for this song and the memory you retrieved for the previous song. $1=$ not related at all, $4=$ very highly related *For example, if two songs in a row make you think of running, that second memory would have a high relation rating (4). However, if the second song evoked a memory of driving in your car, it would be less related ( 1 or 2 )

Recency: How long ago did this event occur? 1=more than 10 years ago, 2= 5-10 years ago, $3=1-5$ years ago, $4=$ less than a year ago

\section{Specificity:}

$1=$ The toplevel of this system- lifetime period knowledge. Lifetime period knowledge encompasses all of the information pertaining to a period in your life. This level includes abstract knowledge about the time period, but no events or activities. It is often 
the case that a song will make you think of the time of your life when you listened to that song frequently or when it was very popular.

$2=$ Below lifetime periods- general event knowledge. This level involves memory for events and activities. However, these events extend beyond a single day, either as an extend event (such as a week-long vacation) or a repeated event (such as an weekly activity). For instance, you may listen to a song whenever you go running, drive to school, etc. Hearing the song may make you think "I had this song on a CD that I listened to in the car everyday on the way to high school."

$3=$ The most specific level- event specific knowledge. Memories from this level contain details specific to a single instance of an event that took place in a particular place and time. For example, if you told a story about your move-in day at college, you would talk about who was there, what they were doing, what your room looked like, etc. These details identify the event as unique- distinct from other "moving in" events that may have been similar. You may hear a song and remember the first time you heard it, a time when it had particular meaning, etc. 


\section{Music Begins}

a)

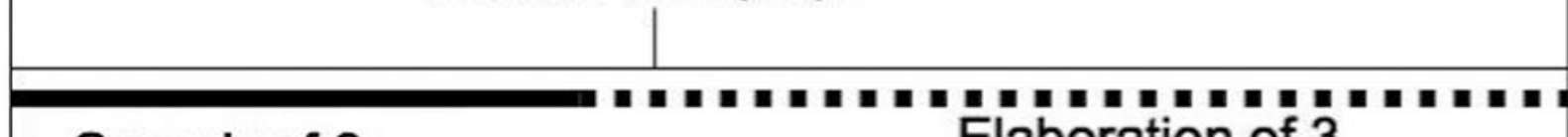

Search of 3

Elaboration of 3

b)

Search

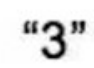

of 3

Figure 1.

Representation of memory phases in a) trials where participants identified only one level of memory and b) trials where participants retrieved memories that included multiple level of memory specificity. Note that in the current study, a button press of 3 indicates event specific memory and a button press of 1 indicates lifetime period memory. 


\section{Figure 2}
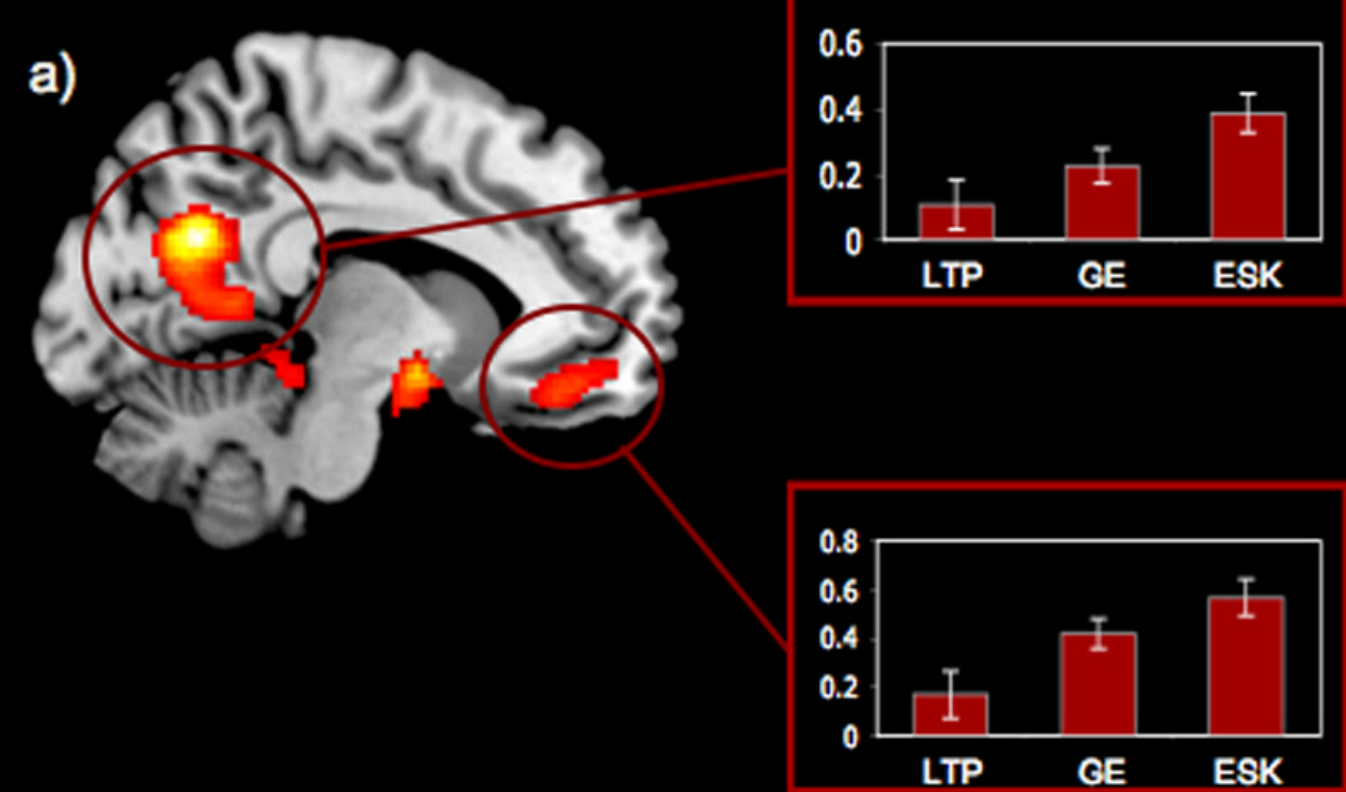

b)

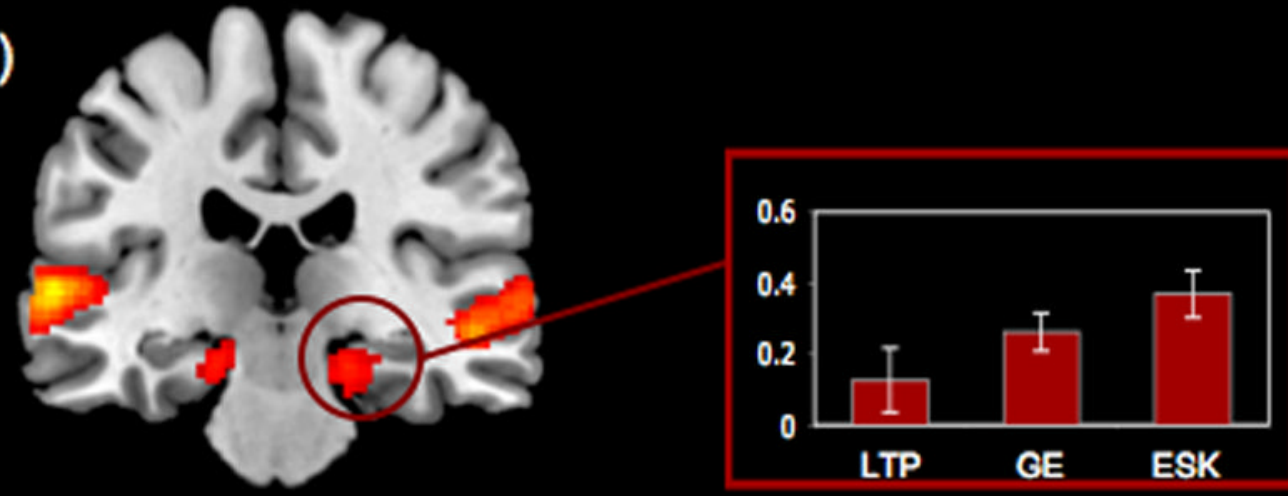

$p<.001, \mathrm{k}=5$

Figure 2.

Regions of activation (at $\mathrm{p}<.001, \mathrm{k} \geq 5$ ) associated with retrieval of all autobiographical memories included: $a$ ) posterior cingulate $(x=-10, y=-60, z=26)$ and ventromedial prefrontal cortex ( $x=-2, y=58, z=-10)$, and $b)$ right parahippocampal gyrus $(x=18, y=-24$, $z=-16)$. 


\section{Figure 3}
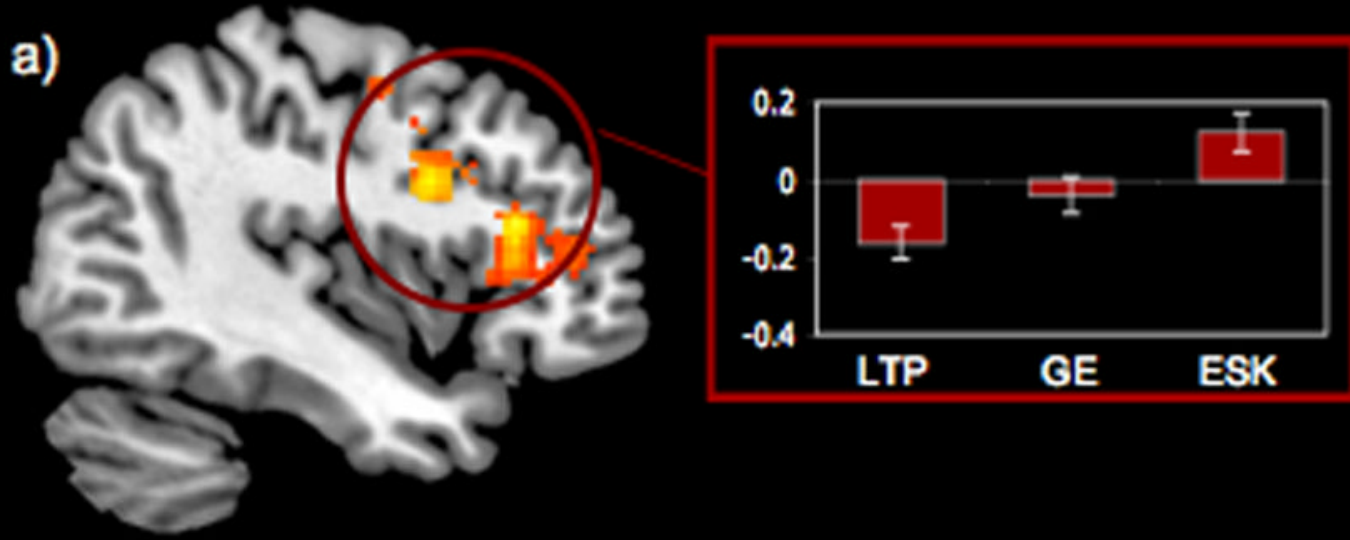

b)

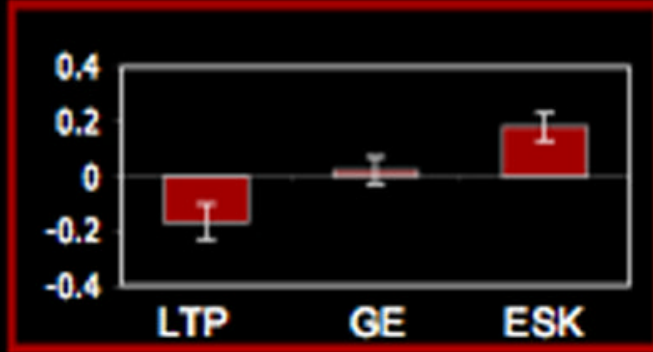

$p<.001, k=5$

Figure 3.

Regions of activation (at $\mathrm{p}<.001, \mathrm{k} \geq 5$ ) associated with retrieval of event specific and general event memories greater than lifetime period memories included: $a$ ) left dorsolateral prefrontal cortex $(x=-42, y=28, z=18)$ and $b)$ dorsomedial prefrontal cortex $(x=-4, y=8$, $z=56)$. 


\section{Figure 4}
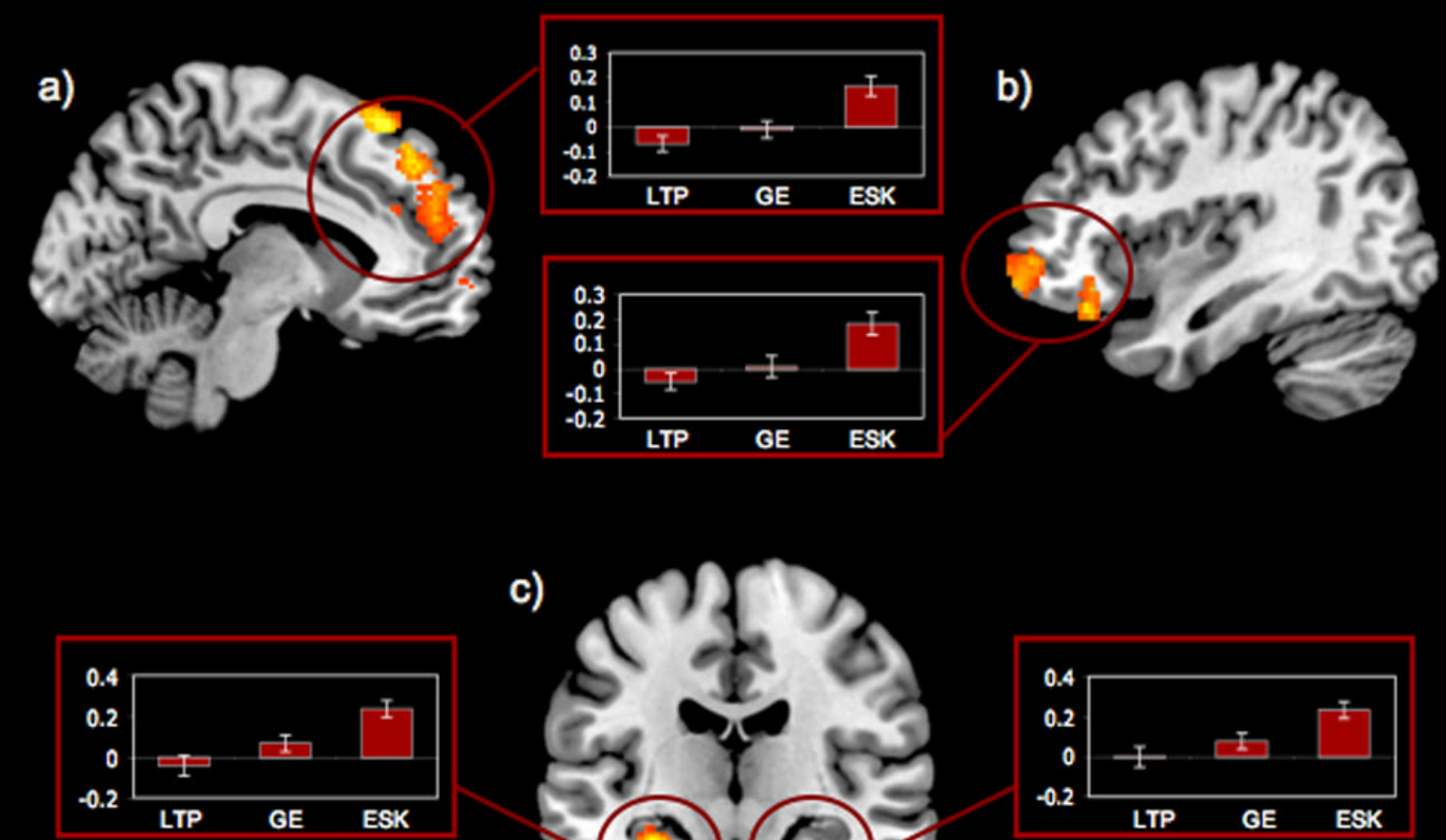

c)
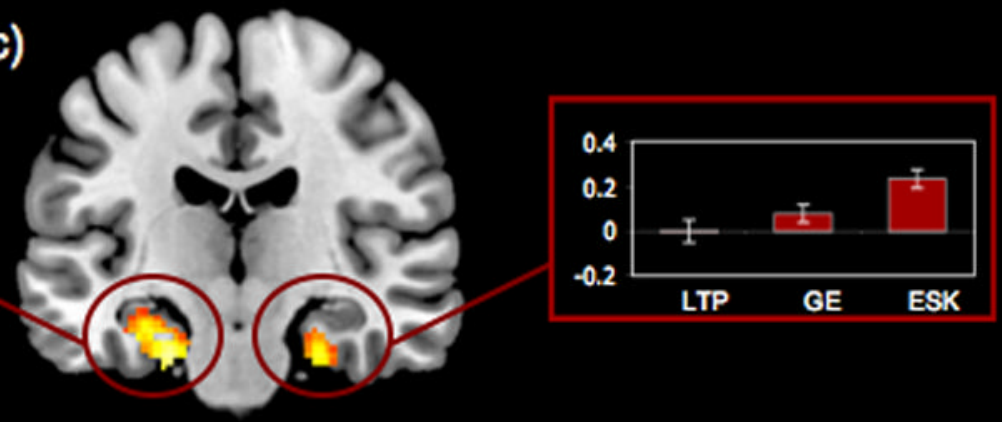

Figure 4.

Regions of activation (at $\mathrm{p}<.001, \mathrm{k} \geq 5$ ) associated with retrieval of event specific greater than general event memories included $a)$ dorsomedial prefrontal cortex $(x=-8, y=12, z=46)$, $b$ ) ventrolateral prefrontal cortex $(x=-60, y=16, z=4)$, and $c)$ bilateral medial temporal lobe $(x=-22, y=-14, z=-30$ and $x=26, y=-6, z=-38)$. 


\section{Figure 5}

a)

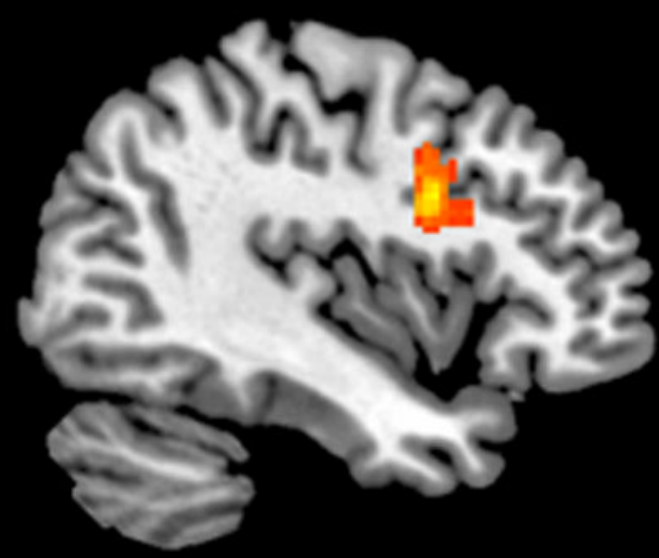

b)

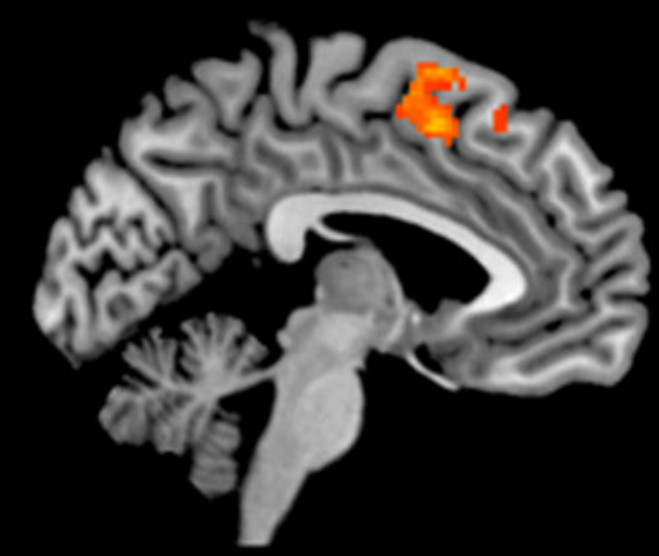

Figure 5.

Regions of activation (at $\mathrm{p}<.001, \mathrm{k} \geq 5$ ) associated with a continual increase in specificity (event specific > general event > lifetime period) included: $a$ ) dorsolateral $(x=-42, y=8$, $z=28)$ and $b$ ) dorsomedial $(x=-6, y=10, z=52)$ prefrontal cortex. 


\section{Table 1}

Summary of Behavioral Data

\begin{tabular}{lccc} 
& \multicolumn{3}{c}{ Average Memory Rating } \\
& Lifetime Period & General Event & Event Specific \\
\cline { 2 - 4 } Emotional Valence & $2.33(.66)$ & $2.57(.52)$ & $2.78(.57)^{*+}$ \\
Emotional Intensity & $2.15(.79)$ & $2.26(.57)$ & $2.59(.69)^{\wedge}$ \\
Re-experiencing & $2.18(.60)$ & $2.60(.34)$ & $3.04(.39)^{* \wedge_{+}}$ \\
Vividness & $2.32(.69)$ & $2.76(.43)$ & $3.21(.41)^{* \wedge_{+}}$ \\
\hline
\end{tabular}

Ratings on a 1-4 scale (low to high); For Emotional Valence, $1=$ Negative and 4=Positive

Standard deviations are in parentheses

General Event significantly greater than Lifetime Period, $p<.05$

$\hat{E v e n t ~ S p e c i f i c ~ s i g n i f i c a n t l y ~ g r e a t e r ~ t h a n ~ G e n e r a l ~ E v e n t, ~} p<.05$

${ }^{+}$Event Specific significantly greater than Lifetime Period, $p<.05$ 


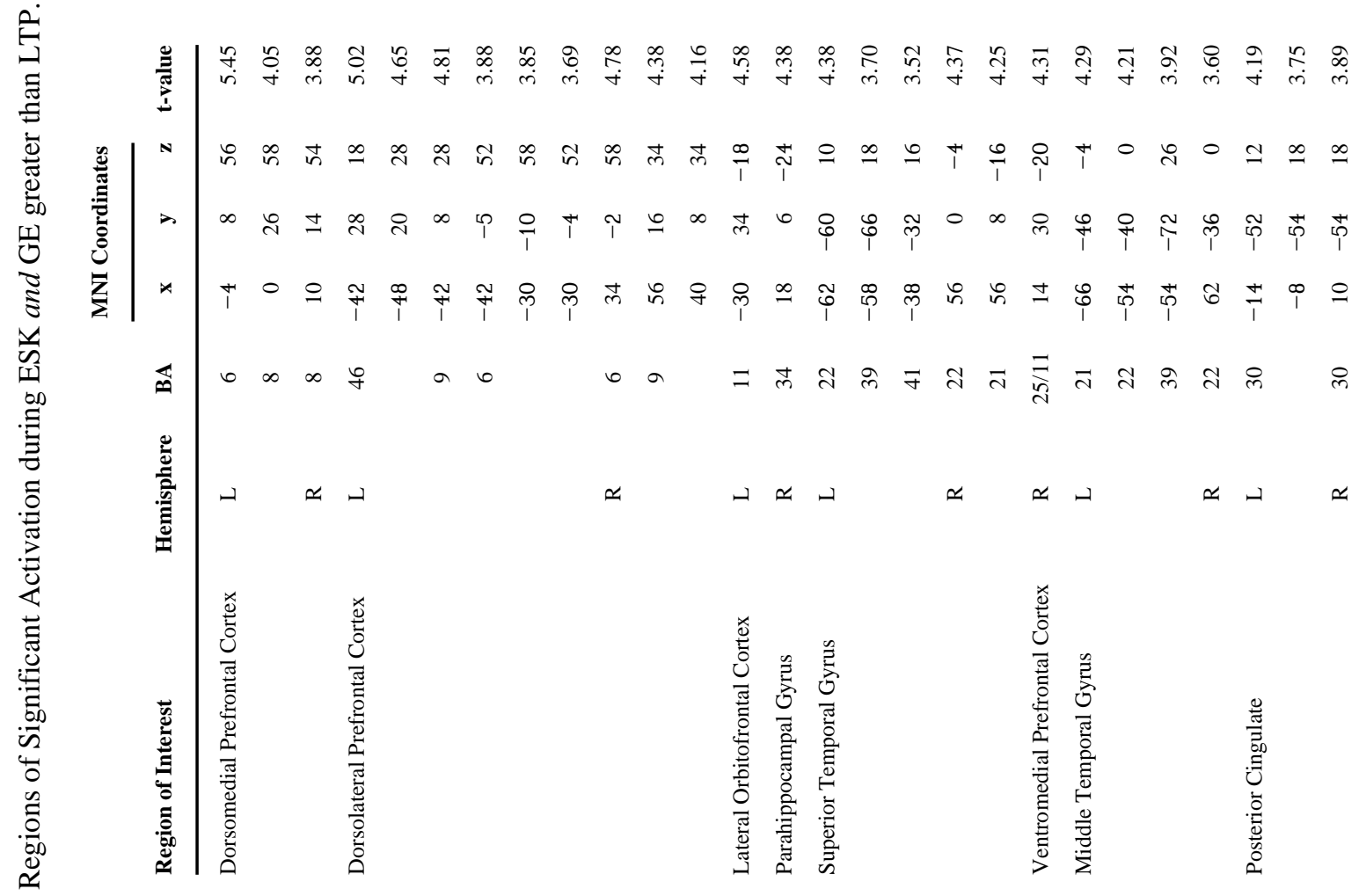




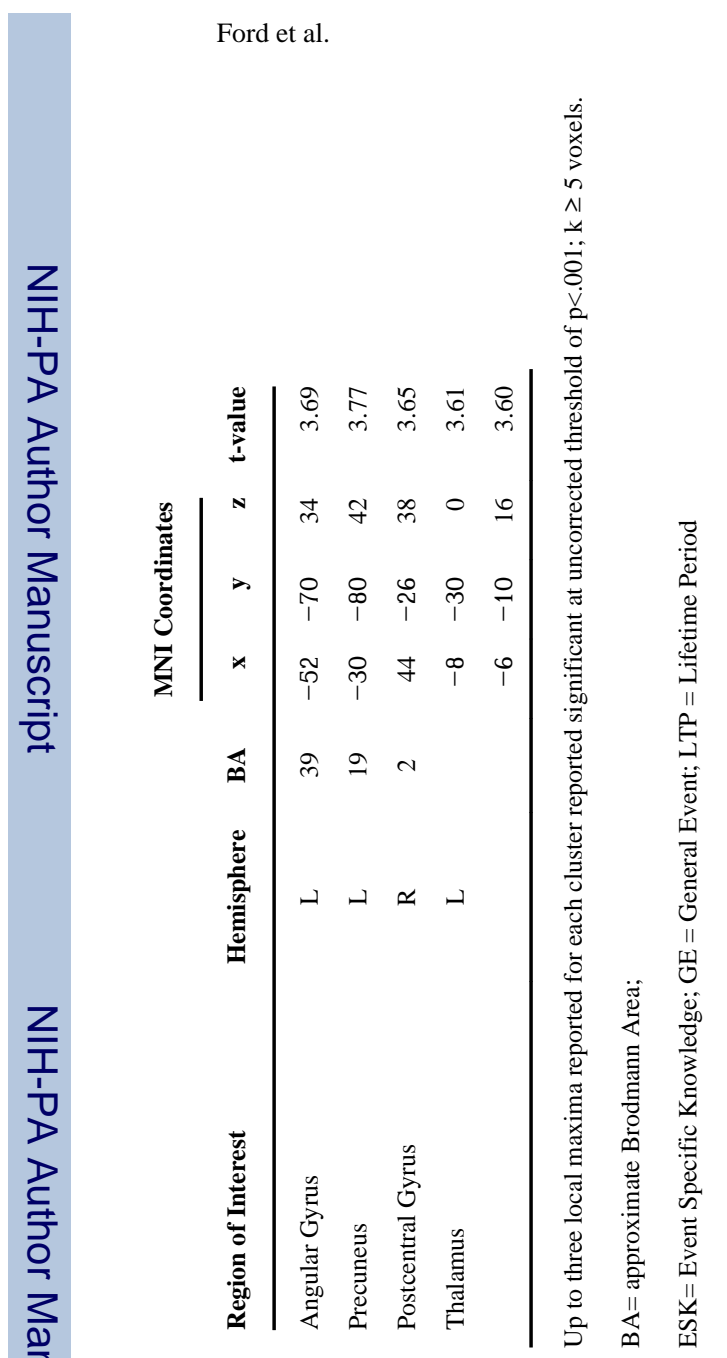

Page 25 


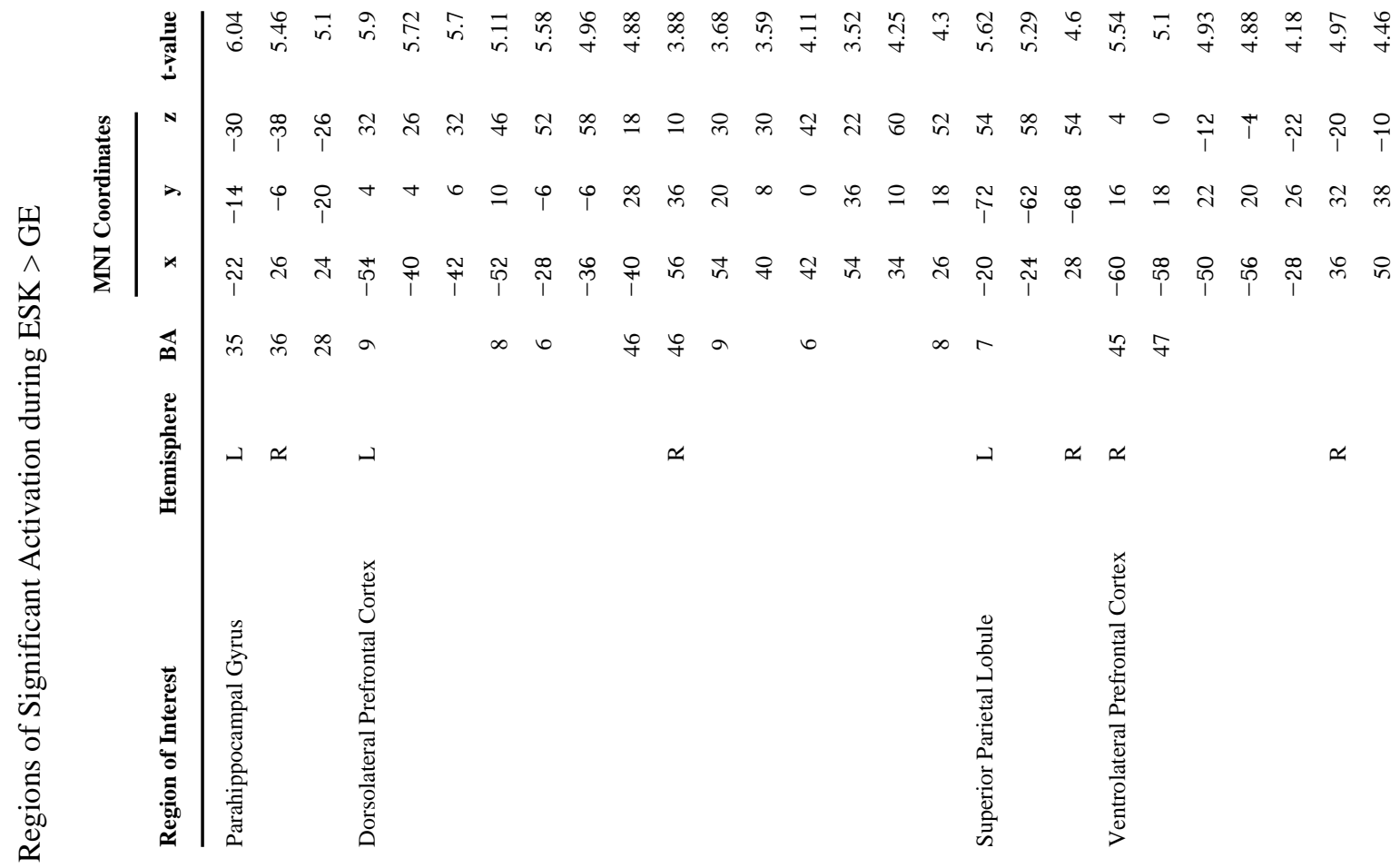




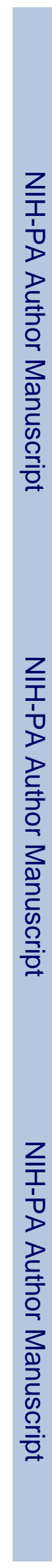

Ford et al.

Page 27

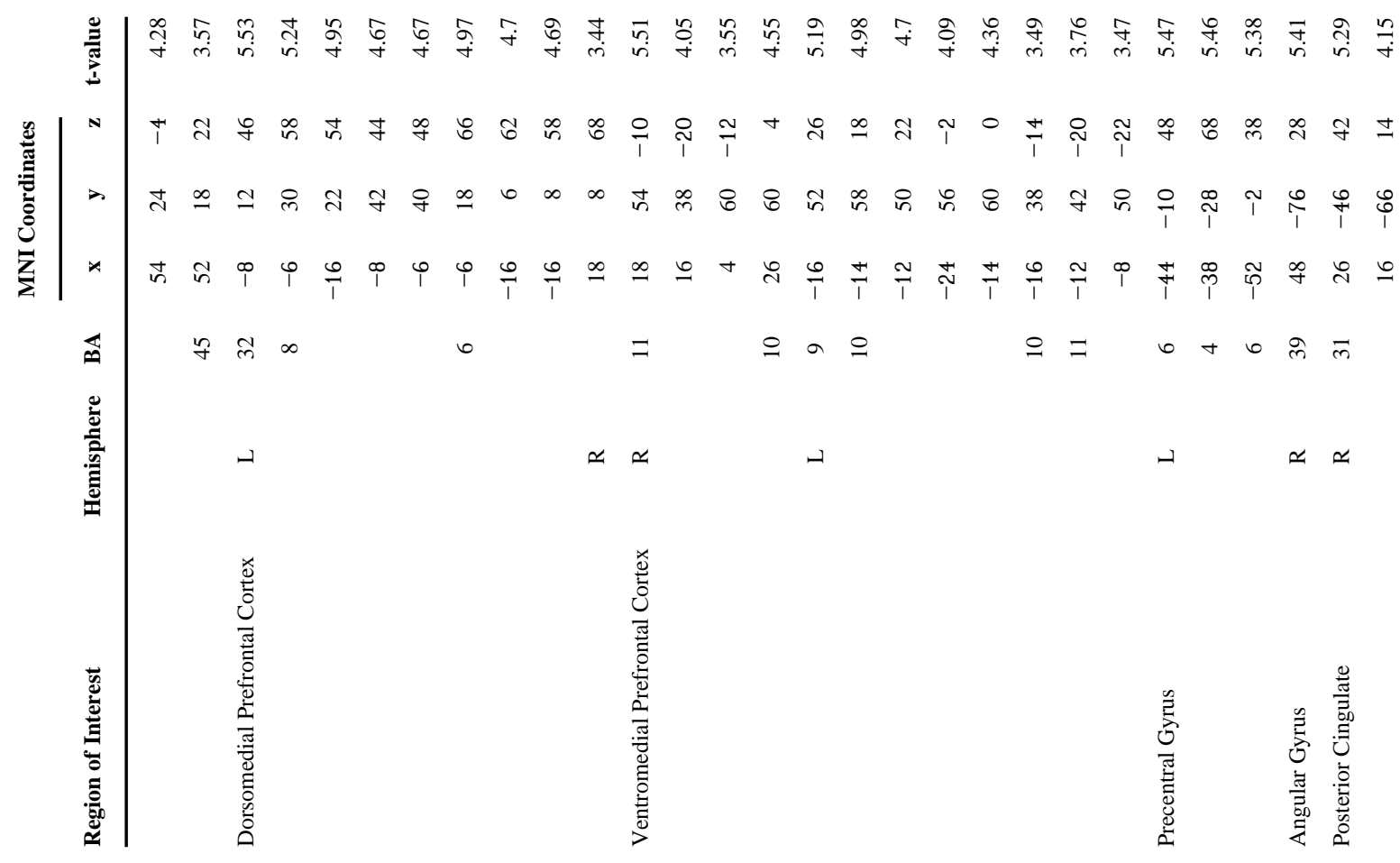

Neuropsychologia. Author manuscript; available in PMC 2012 July 1. 


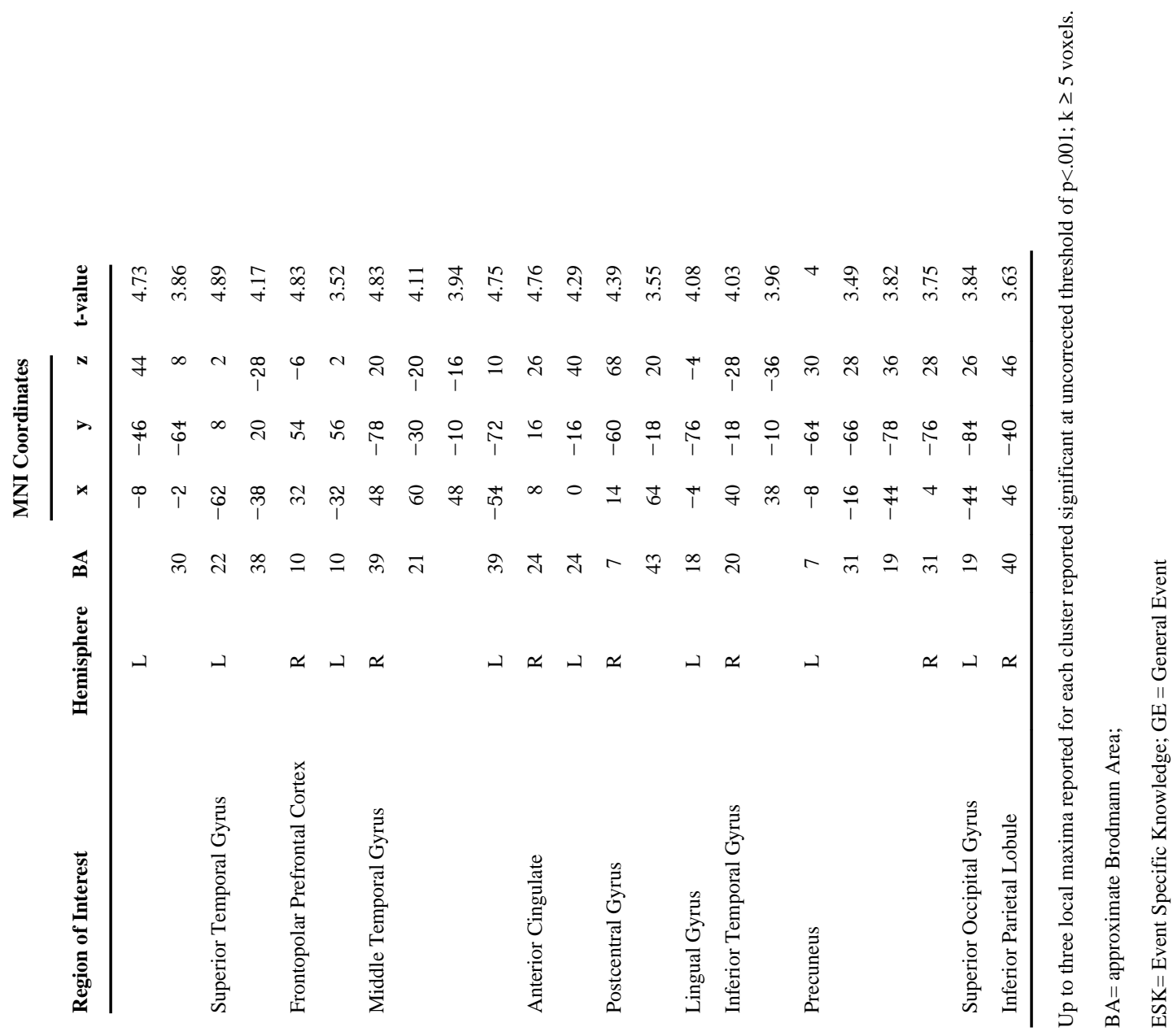




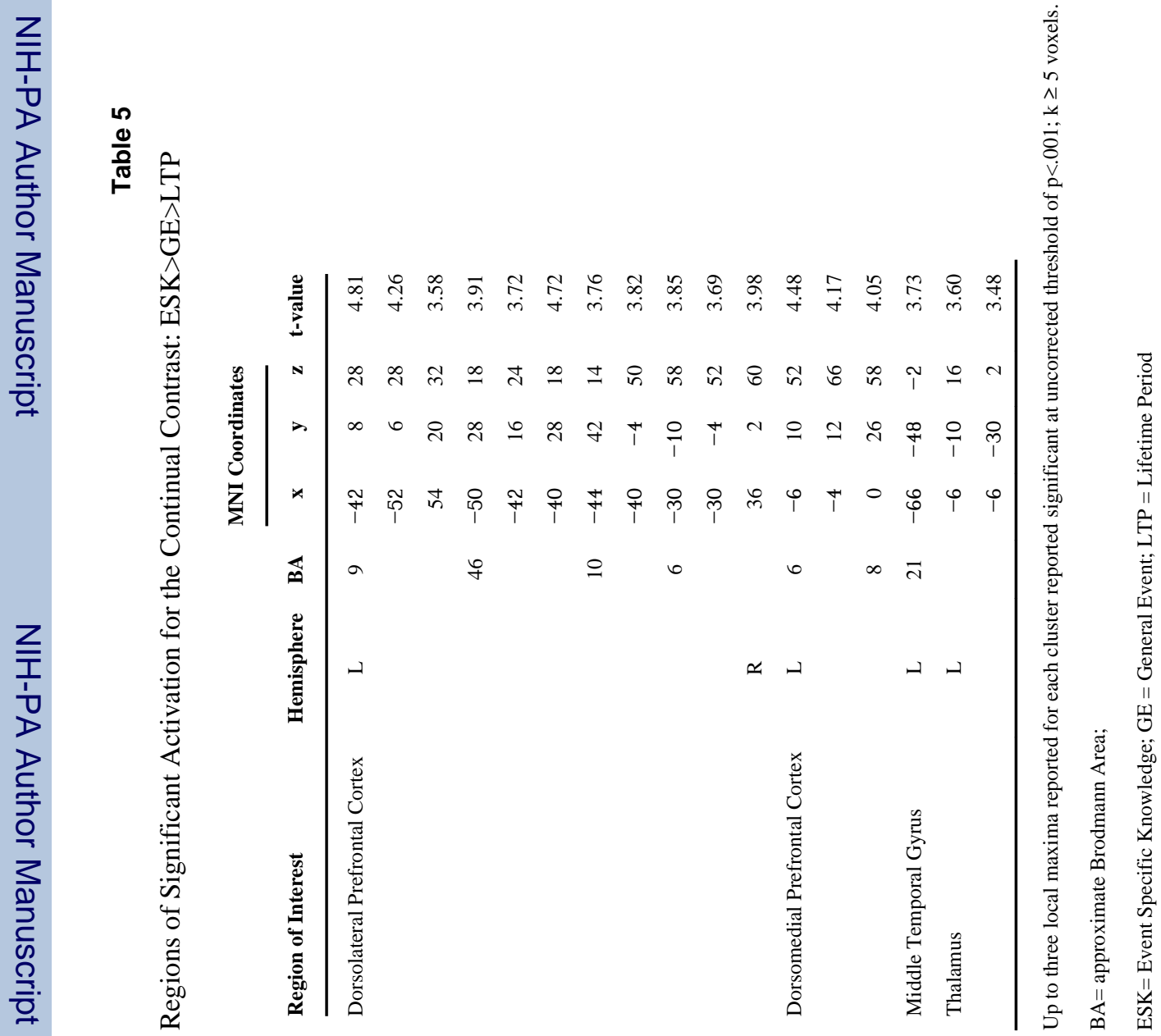

Neuropsychologia. Author manuscript; available in PMC 2012 July 1. 